\title{
A Study of Relationship of Sleep Apnoea with Type-2 Dm in Middle Aged Males of Eastern Region of Bihar
}

\author{
Dr Narayan Sinha ${ }^{1}$, Dr Arohi Abhinav Jayaswal', \\ ${ }^{1}$ HOD, Dept. Of Physiology JLN Medical college Bhagalpur \\ ${ }^{2}$ Tutor, Dept. Of Physiology JLN Medical College Bhagalpur
}

\section{Introduction}

The widely accepted definition of obstructive sleep apnoea (OSA) is a clinical condition in which there is intermittent and repeated upper airway collapse during sleep. This results in irregular breathing at night and excessive sleepiness during the day. Complete apnoea is defined as a ten-second pause in breathing activity. Partial apnoea, also known as hypopnoea, is characterised by a ten-second period in which ventilation is reduced by at least $50 \%$.

Risk factors include: Obesity (strongest risk factor), Male gender, Middle age (55-59 in men, 60-64 in women), Smoking, Sedative drugs, Excess alcohol consumption, Possibly genetic tendency related to jaw morphology.

Type 2 diabetes mellitus (DM) and obstructive sleep apnea (OSA) share several clinical findings: obesity, hypertension, and impaired glucose tolerance. OSA may be an under-recognized comorbidity of DM. Unsurprisingly, the disorders commonly coexist.(1) Mechanistically, OSA may aggravate Insulin Resistance (IR) and type 2 diabetes via intermittent hypoxia, fragmented sleep and elevated sympathetic activity. Diabetes may contribute to OSA via neuropathy and weight gain related to insulin use. Most cross-sectional studies have demonstrated that OSA is independently associated with IR and type 2 diabetes in adult sleep clinic populations and in unselected communities, independent of age and BMI, but prospective incidence studies have been less convincing.(1)

This is the first study of its kind done in this region of Bihar. The purpose of this study is to estimate the proportion of middle aged male patients with type 2 DM at high risk for Sleep Apnoea and whether there is any significant relationship between the two in the middle aged males of the eastern region of Bihar.

\section{Epidemiology}

OSA is a worldwide phenomenon. Studies suggest a prevalence in Western countries of $3-7 \%$ of middle-aged men and $2-5 \%$ of middle-aged women. The prevalence of OSA in patients with type 2 diabetes has been reported to vary between $23 \%$ (2) and $86 \%$ (3), with differences in study populations and OSA definitions explaining the marked variation in results(4).

There is evidence that regular snoring and OSA are independently associated with alterations in glucose metabolism. [5] Thus, OSA might be a risk factor for the development of type 2 diabetes.

According to the Centers for Disease Control and Prevention, 25.6 million Americans aged 20 years or older suffer from diabetes, and Type 2 diabetes accounts for about 90 to 95 percent of all diagnosed cases. Seven in 10 people with Type 2 diabetes also have obstructive sleep apnea, and the severity of the sleep disorder directly impacts diabetes symptoms; the more severe a diabetic's untreated sleep apnea, the poorer their glucose control.

\section{Materials and Methods}

This is the first study of its kind done in this region. The study was done at Jawaharlal Nehru Medical College \& Hospital Bhagalpur in male Medicine OPD. The period of study was of 7 months. This Cross sectional study enrolled 240 Male subjects between age group of 55 to 59 years. They were assessed for fasting blood glucose levels. Subjects having fasting blood sugar levels above $126 \mathrm{mg} / \mathrm{dl}$ were categorised as diabetics.(6)

In our study the number of diabetics were 72 . The control group having 168 persons were not diabetic.

The statistical analysis was done using SPSS software package.

DIAGNOSIS OF SLEEP APNOEA : Symptoms such as personality change, episodes of apnoea, irritability and restlessness at night may be better elicited by taking a history from a partner. OSA risk was determined using the Berlin Questionnaire. (7,8) The low risk cases of sleep apnea are those in which if there is only 1 or no categories where the score is positive. The high risk cases of sleep apnea are those in which there are 2 or more categories where the score is positive. 
Daytime sleepiness can also be assessed using the Epworth Sleepiness Scale.(9,10) A score greater than 10 should prompt referral.

\section{EXCLUSION CRITERIA (9)}

Persons having craniofacial or pharyngeal abnormalities like retrognathia, micrognathia, enlarged tonsils, macroglossia, thickening or lengthening of the soft palate or uvula, nasal polyps, rhinitis or any deformity of the nose, Fragmented sleep (decreased quality of sleep), Sleep deprivation (decreased quantity of sleep), Night Shift work, Depression, Narcolepsy, Hypothyroidism, Restless leg syndrome/periodic limb movement disorder, Drugs: Sedatives , Stimulants (caffeine, theophyllines, amfetamines), Beta-blockers, Selective serotonin reuptake inhibitors (SSRIs), Idiopathic hypersomnolence, Excess alcohol, Neurological conditions: Dystrophica myotonica, Previous encephalitis, Previous head injury, Parkinsonism were excluded from the study.

\section{OBSERVATIONS}

\begin{tabular}{|c|c|l|c|l|l|}
\hline & $\begin{array}{l}\text { Total } \\
\text { NO. OF } \\
\text { CASES }\end{array}$ & $\begin{array}{l}\text { NO. OF CASES OF } \\
\text { SLEEP } \\
\text { (BERLIN'S CRITERIA) }\end{array}$ & $\begin{array}{c}\text { FASTING } \\
\text { BLOOD } \\
\text { GLUCOSE+-2SD }\end{array}$ & $\%$ & P \\
\hline DIABETICS & 72 & $\begin{array}{l}\text { HIGH RISK 60 } \\
\text { LOW RISK 12 } \\
\text { HIGH RISK 08 } \\
\text { LOW RISK 160 }\end{array}$ & $162 \pm 12$ & $83.33 \%$ & $<0.01$ \\
$\begin{array}{c}\text { NON- } \\
\text { DIABETICS }\end{array}$ & 168 & & $116 \pm 15$ & $16.66 \%$ & \\
& & & $94 \pm 12$ & $4.76 \%$ & $>0.05$ \\
\end{tabular}

\section{Discussion}

There was positive significant relationship of sleep apnoea with diabetes in middle aged male subjects in our study. 60 out of 72 diabetics were at high risk of sleep apnea (83.33\%). Whereas only 8 out of 168 $(4.76 \%)$ non diabetics were at high risk of sleep apnea. The result of this study coincides with the result of study done by Foster GD et al. (3)

Further there is positive significant relationship between Type 2 diabetes and sleep apnea $(p<0.01)$. There was no significant relationship between normal non diabetic and incidence of sleep anea cases in our study $(\mathrm{p}>0.05)$.

Patients suffering from Type 2 diabetes or hypertension should discuss their risk for obstructive sleep apnea with a board-certified sleep medicine physician, who can oversee their diagnosis and prescribe the most appropriate treatment.

"Treating sleep apnea in diabetics improves nighttime glucose levels and insulin sensitivity," said Badr. "Treatment also will provide benefits of improved sleep unrelated to diabetes - including increased alertness during the day and improved memory and cognitive function."

There are five main management options:

\section{Behavioural interventions}

Lifestyle changes should be discussed: Weight loss is appropriate if obesity is an issue and gastric surgery may be appropriate in some patients who fit the criteria for this type of treatment. There is evidence for improvement of OSA symptoms with weight loss in mild OSA,(11) but more research is needed.

Smoking cessation should be advocated from a general health perspective; however, again there is no guarantee that this will alleviate OSA symptoms.

Alcohol should be avoided in the evening, as should sedative and hypnotic medication, as these all decrease airway dilator function. 


\title{
2.CPAP
}

This acts as a pneumatic splint, maintaining upper airway patency. A flow generator delivers pressure through air tubing to a nasal or facial mask worn overnight. Most patients require lifelong therapy. CPAP is a wellestablished treatment with a solid evidence base, most suitable for patients with moderate to-severe OSA. CPAP is currently recommended as a treatment option for adults with mild OSAHS if they have symptoms that affect their quality of life and ability to go about their daily activities and lifestyle advice and any other relevant treatment options have been unsuccessful or are considered inappropriate.(12) A recent study from the University of Chicago shows that continuous positive airway pressure (CPAP) treatment of sleep apnea may have as much of an effect as prescribed oral diabetes medications. "In our study, one week of optimal CPAP use lowered average 24-hour glucose levels and improved post-breakfast glucose response in Type 2 diabetics with obstructive sleep apnea," said Esra Tasali, MD, lead author of the study and assistant professor of medicine, pulmonary and critical care medicine at the University of Chicago Department of Medicine. "The dawn phenomenon, an early-morning increase in blood sugar in people who have Type 2 diabetes, also was reduced by 45 percent as a result of CPAP therapy."

\section{Intra-oral devices}

Current evidence on soft-palate implants for obstructive sleep apnoea (OSA) raises no major safety concerns, but there is inadequate evidence that soft-palate implants are efficacious in the treatment of obstructive sleep apnoea. NICE recommends that soft-palate implants should not be used in the treatment of this condition.(13)

\section{Pharmacological treatments}

The role of pharmacological agents is limited. Modafinil may afford some benefit in patients with daytime sleepiness who are compliant with CPAP treatment, but longer-term studies are needed. The European Respiratory Society have stated that drugs, nasal dilators and apnoea triggered muscle

stimulation cannot be recommended as effective treatments of OSA at the moment.(14)

\author{
5.Surgery \\ The evidence base is at best equivocal. \\ The procedures evaluated include: \\ Uvulopalatopharyngoplasty (UPPP) - patients may be unable to use CPAP subsequently. \\ Laser-assisted uvulopalatopharyngoplasty (LAUP). \\ Mandibular advancement. \\ Maxillary advancement. \\ Tonsillectomy - appropriate for tonsillar enlargement. \\ Tracheostomy - may be necessary in severe OSA where other treatments fail.
}

Various techniques have been tried; however, assessment is difficult due to inconsistency of trial methodology.

\section{Complications}

Excessive daytime sleepiness may cause accidents in the home, at work and whilst driving. Irritability, depression and other psychological consequences may ensue. Cardiovascular complications include hypertension, coronary artery disease, congestive cardiac failure. OSA has also been identified as an independent risk factor for stroke and all-cause mortality.(15) There is also an increased risk of cor pulmonale, type 2 respiratory failure, metabolic disturbances, such as insulin resistance in patients with OSA.(16)

\section{Prognosis}

Untreated OSA can lead to significant neurocognitive and also cardiovascular morbidity. The cognitive impairment can lead to reduced concentration, accidents and also memory problems. For patients who respond to CPAP, the short-term prognosis is excellent. Placebo-controlled studies have shown a positive benefit in terms of reduction in daytime sleepiness and in snoring, and an improvement in cognitive function and general health status after 4-8 weeks of treatment. Treatment can significantly decrease cardiovascular complications, especially in those patients with severe OSA.

\section{Conclusion}

There was positive significant relationship of diabetes with sleep apnea in our study. Further study is needed to be done to demonstrate the correlation of severity of sleep apnoea with the level of fasting blood glucose. 


\section{References}

[1]. Garun S Hamilton and Matthew T Naughton. Impact of obstructive sleep apnoea on diabetes and cardiovascular Disease. Med J Aust 2013; 199 (8): 27-30.

[2]. West SD, Nicoll DJ, Stradling JR. Prevalence of obstructive sleep apnoea in men with type 2 diabetes. Thorax 2006; 61: 945-950.

[3]. Foster GD, Sanders MH, Millman R, et al. Obstructive sleep apnea among obese patients with type 2 diabetes. Diabetes Care 2009; 32: 1017-1019.

[4]. Iftikhar IH, Hays ER, Iverson MA, et al. Effect of oral appliances on bloo pressure in obstructive sleep apnea: a systematic review and meta-analysis. J Clin Sleep Med 2013; 9: 165-174.

[5]. Obstructive sleep apnea and type 2 diabetes; Eur J Med Res. 2010 Nov 4;15 Suppl 2:152-6.

[6]. Harrison's Principles of Internal Medicine. 2013;

[7]. Cass AR, Alonso WJ, Islam J, Weller SC .Risk of obstructive sleep apnea in patients with type 2 diabetes mellitus. Fam Med. 2013 Jul- Aug;45(7):492-500. Netzer NC, Stoohs RA, Netzer CM, Clark K, Strohl KP Using Berlin

[8]. Questionnaire to identify patients at risk for the sleep apnea syndrome. Ann Internal Med. 1999 Oct 5; 131(7):485-91.

[9]. Management of obstructive sleep apnoea/hypopnea syndrome in adults, Scottish Intercollegiate Guidelines Network - SIGN (2003).

[10]. Epworth Sleepiness Scale, British Snoring \& Sleep Apnoea Association

[11]. Tuomilehto HP, Seppa JM, Partinen MM, et al; Lifestyle intervention with weight reduction: first-line treatment in mild Am J Respir Crit Care Med. 2009 Feb 15;179(4):320-7. Epub 2008 Nov 14.

[12]. Sleep apnoea - Continuous positive airway pressure for the treatment of obstructive sleep apnoea/hypopnoea syndrome; NICE Technology Appraisal (March 2008)

[13]. Soft-palate implants for obstructive sleep apnoea, NICE Interventional Procedure Guideline (November 2007)

[14]. Non-CPAP therapies in obstructive sleep apnoea, European Respiratory Society (2011)

[15]. Obstructive sleep apnea and stroke; Expert Rev Cardiovasc Ther. 2012 Apr,10(4):525-35

[16]. Is obstructive sleep apnea a risk factor for diabetes?; Discov Med. 2011 Jul12(62):17-24. 